\title{
Composite structural modeling and tensile mechanical behavior of graphene reinforced metal matrix composites
}

\author{
Yishi Su, Zan Li, Yang Yu, Lei Zhao, Zhiqiang Li, Qiang Guo, Dingbang Xiong and Di Zhang*
}

\begin{abstract}
Owing to its distinguished mechanical stiffness and strength, graphene has become an ideal reinforcing material in kinds of composite materials. In this work, the graphene (reduced graphene oxide) reinforced aluminum (Al) matrix composites were fabricated by flaky powder metallurgy. Tensile tests of pure Al matrix and graphene/Al composites with bioinspired layered structures are conducted. By means of an independently developed Python-based structural modeling program, three-dimensional microscopic structural models of graphene/Al composites can be established, in which the size, shape, orientation, location and content of graphene can be reconstructed in line with the actual graphene/Al composite structures. Elastoplastic mechanical properties, damaged materials behaviors, grapheneAl interfacial behaviors and reasonable boundary conditions are introduced and applied to perform the simulations. Based on the experimental and numerical tensile behaviors of graphene/Al composites, the effects of graphene morphology, graphene-Al interface, composite configuration and failure behavior within the tensile mechanical deformations of graphene/Al composites can be revealed and indicated, respectively. From the analysis above, a good understanding can be brought to light for the deformation mechanism of graphene/Al composites.
\end{abstract}

Keywords: graphene/Al composites, structural modeling, mechanical properties, composite configuration, failure behavior

\section{INTRODUCTION}

Graphene, as a perfect two-dimensional (2D) carbon atomic material, has attracted a lot of attentions due to its excellent mechanical and functional properties [1-3], such as high mechanical stiffness (1 TPa) [4], high fracture strength $(130 \mathrm{GPa})$ [5], high thermal conductivity $\left(3 \times 10^{3} \mathrm{~W} \mathrm{~m}^{-1} \mathrm{~K}^{-1}\right)$ [6] and super electron mobility $\left(2 \times 10^{5} \mathrm{~cm}^{2} \mathrm{~V}^{-1} \mathrm{~s}^{-1}\right)$ [7]. Among these properties, the high mechanical stiffness and strength make graphene owning an outstanding potential as the reinforcing material in all kinds of composites [8-10]. In comparison to the graphene reinforcement, a lot of researchers have focused on synthesizing the composites of graphene oxide (GO) in polymer [11,12] or metal [13-15] matrix in the past forty years. Due to its lower mechanical properties and nonuniform dispersion [16,17], GO reinforcement distributed in the polymer or metal matrix could result in a poor enhancing effect on the mechanical properties of GO-based composites [12,14]. Although the mechanical properties of GO are much lower than pristine graphene, the GO can be reduced as graphene (reduced graphene oxide, RGO) that possesses larger mechanical properties similar to those of graphene $[18,19]$. Previous experimental measurements showed a critical stress of $130 \mathrm{GPa}$, and a strain of about $25 \%$ for a graphene membrane, while the Young modulus was obtained to be roughly 1 TPa $[2,5]$. Moreover, the elastic and mechanical properties of graphene or GO have been computed via different computational and theoretical approaches, such as the density functional theory (DFT), quantum mechanicalbased methods, molecular dynamics (MD) simulation, and continuum mechanics-based methods [16,20]. Young's modulus of GO relates to the number of layers: the measured Young's modulus of single-layer, doublelayer and triple-layer GO membranes were 223 $\pm 17.7,444$ \pm 25.3 and $665 \pm 34.6 \mathrm{GPa}$, respectively [21]. The mechanical properties of GO were examined and compared to those of graphene. Although significantly weaker in tensile deformation than graphene (fracture stress = $116 \mathrm{GPa}$ ), GO (fracture stress $=63 \mathrm{GPa}$ ) potentially has great strength provided it does not contain large holes [22]. Meanwhile, just a few simulating studies have been

State Key Laboratory of Metal Matrix Composites, Shanghai Jiao Tong University, Shanghai 200240, China

* Corresponding author (email: zhangdi@sjtu.edu.cn) 
done to reveal the relationship between the composite structure and mechanical property of the graphene-based polymer matrix composites. Within the traditional theoretical framework, mechanical properties of graphenebased composites were studied by constructing a threedimensional (3D) representative volume element (RVE) model composed of a cubic portion of the matrix containing the plate model of graphene in center [20]. On the 3D RVE-based multiscale structural model, the graphene sheets and polymer matrix were modeled as continuums, and the interfacial property and fracture behavior were also studied [23]. Moreover, the graphene was also modeled as circular plates and a series of microscopic structural models of graphene-based polymer matrix composites were generated. The graphene morphologies (e.g. the orientation, clustering, exfoliated or intercalated, aspect ratio, etc.) were taken into account during the model generation process $[24,25]$. In comparison, there have been few reports on the relationship between the composite structure and mechanical property of graphene/Al composites, while the main challenge lies in how to reveal the graphene reinforcing effect of graphene/ Al composites without destroying its physical structure. Therefore, it may still be a long way to go until the mechanical properties of graphene/Al composites can be improved and developed well in engineering applications.

By means of a novel flaky powder metallurgy technique, the graphene/Al composites with bioinspired layered structure were fabricated and the graphene could produce a significant enhancing effect on their mechanical properties. Statistic distributions of graphene in-plane size and Al layer thickness were determined from microscopic structures. Based on the independently developed Python-based structural modeling program, the 3D microscopic structural models of graphene/Al composites can be established, where the size, shape, orientation, location and content of graphene are modeled in line with the actual graphene/Al composites. Elastoplastic mechanical property, graphene-Al interfacial behavior, damaged materials behavior and reasonable boundary conditions are introduced into the simulations. Numerical and experimental tensile mechanical deformations of graphene/ $\mathrm{Al}$ composites are carried out and verified to reveal the strengthening effect of graphene on the mechanical behavior of graphene/Al composites with different graphene content. At the same time, the effects of graphene morphology, interfacial behavior, composite configuration and failure behavior on the mechanical behavior of graphene/Al composites are also indicated.

\section{EXPERIMENTAL SECTION}

In this work, a novel flaky powder metallurgy route was applied to prepare the graphene/Al composites with bioinspired layered structures, which has been proved to be effective to fabricate the carbon nanotubes reinforced aluminum composites in our previous work $[26,27]$. In this route, the GO (95\% in purity) nanosheets modified with hydroxyl or carboxyl groups on their surface rather than the graphene were applied as the raw material. Meanwhile, the GO nanosheets were dispersed in deionized water to form stable solutions, which were ultrasonicated to exfoliate the GO into few-layer nanosheets. $\mathrm{Al}$ spherical powders $(\sim 10 \mu \mathrm{m}$ in diameter and $99.99 \%$ in purity) were ball-milled into Al flakes with an initial thickness of $250 \pm 32 \mathrm{~nm}$ in a stainless steel attritor at 325 rpm (Fig. 1a). Al flakes were further dispersed with GO in pure ethanol to form a powder slurry. The mixed powder slurry was stirred, filtered and rinsed several times with pure ethanol to obtain the $\mathrm{GO} / \mathrm{Al}$ composite powders (Fig. 1b), which were stirred at $400 \mathrm{rpm}$ and dried at $333 \mathrm{~K}$ for $24 \mathrm{~h}$ in vacuum. The GO could be sufficiently reduced to graphene through annealing in a tube furnace at $773 \mathrm{~K}$ for $2 \mathrm{~h}$, in which a $\mathrm{H}_{2}$-Ar mixed gas flow ( $5 \mathrm{vol} \%$ $\mathrm{H}_{2}$ and $95 \mathrm{vol} \% \mathrm{Ar}$ ) with a flow rate of $40 \mathrm{~mL} \mathrm{~min}^{-1}$. RGO/Al composite powders were compacted into cylindrical billets of $40 \mathrm{~mm}$ in diameter and $30 \mathrm{~mm}$ in height under a static pressure of $500 \mathrm{MPa}$, and then were consolidated by vacuum hot-pressed at $803 \mathrm{~K}$ for $1 \mathrm{~h}$. For comparison, $\mathrm{Al}$ matrix samples were also produced just using the ball-milled Al flakes. Microstructural characterizations of dispersed $\mathrm{GO}$ on the surfaces of $\mathrm{Al}$ flakes and the fabricated graphene/ $\mathrm{Al}$ composites were obtained by transmission electron microscopy (TEM, JEOL JEM2010), in which the Al layers with an average thickness of $h=215 \mathrm{~nm}$ can be determined from the statistic results of the graphene/Al composite products, as seen in Fig. 1c. Uniaxial plate tensile specimens with the width of $2 \mathrm{~mm}$, the thickness of $1 \mathrm{~mm}$ and the length of $10 \mathrm{~mm}$ in gauge area were machined from the $\mathrm{Al}$ matrix and graphene/ $\mathrm{Al}$ composites. Uniaxial tensile tests were performed on Shimadzu Autograph AG-I (50 KN) at a constant strain rate of $1 \times 10^{-4} \mathrm{~s}^{-1}$ at room temperature (shown in Fig. 1d).

\section{Numerical procedure}

For systemically studying the relationship between the composite structure and mechanical property of graphene/ $\mathrm{Al}$ composites, a series of $3 \mathrm{D}$ microscopic structural models of graphene/Al composites have been established similar to their actual microstructures. In this study, a Python-based structural modeling program has 

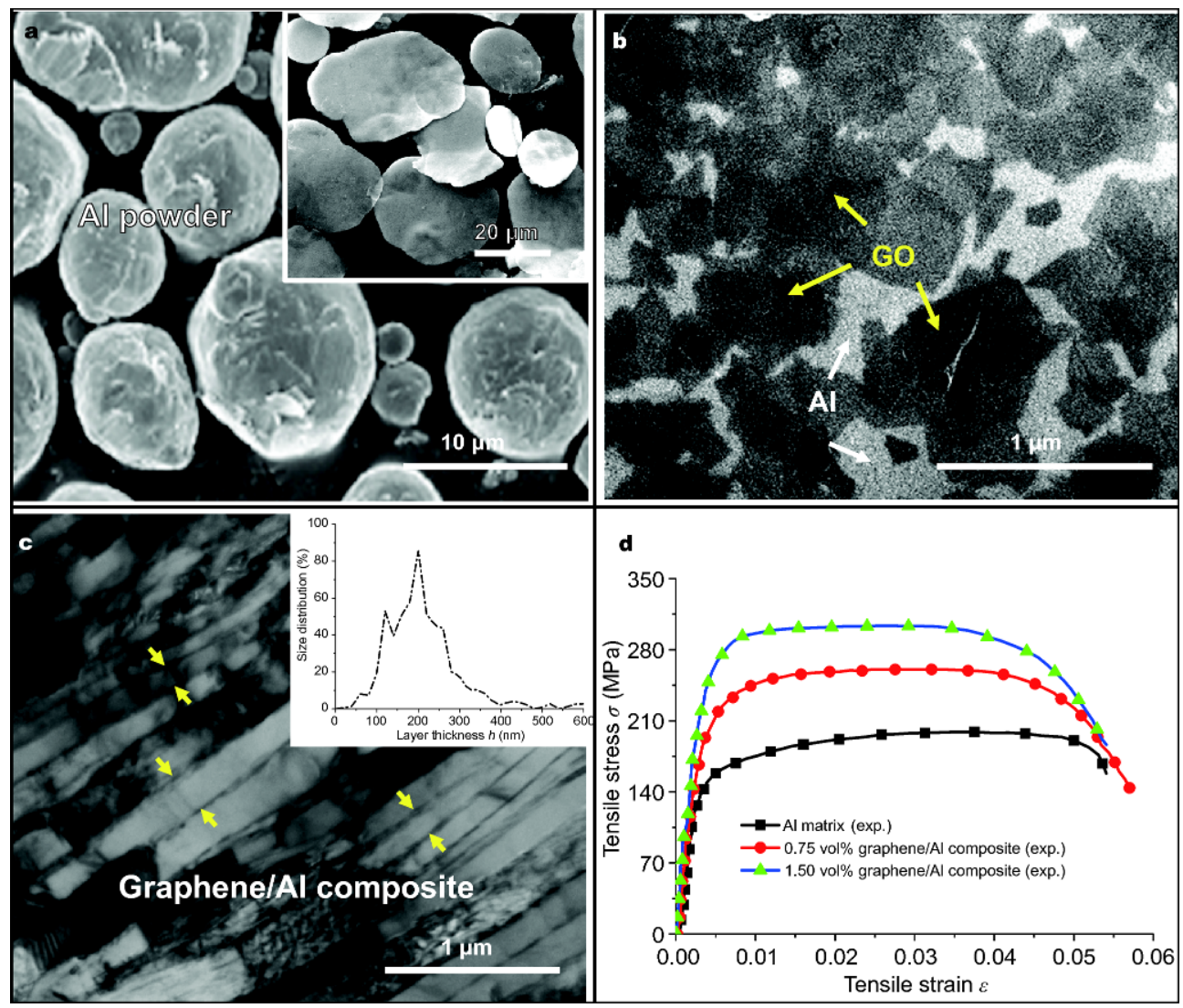

Figure 1 Microstructural characterization and mechanical properties of graphene/Al composites: (a) spherical Al powders; (b) dispersed GO (graphene oxide) on $\mathrm{Al}$ flakes; (c) $1.5 \mathrm{vol} \%$ graphene/ $\mathrm{Al}$ composite with layered structures and (d) tensile stress-strain relations of $\mathrm{Al}$ matrix and graphene /Al composites.

been independently developed, and it can be applied to create the 3D microscopic structural models of graphenebased composites (i.e., high aspect ratio, thin thickness, irregular shape, various orientation and layered structure, etc.).

\section{Structural modeling of graphene and graphene/Al composites}

In Fig. 1b, GO nanosheets were uniformly dispersed on the surfaces of $\mathrm{Al}$ flakes, while the $\mathrm{GO}$ or graphene can usually be treated as $2 \mathrm{D}$ materials. Therefore, the in-plane size distribution of GO is a key factor to create the $3 \mathrm{D}$ microscopic structural models of graphene. In order to evaluate the size distribution of GO or graphene, a statistical in-plane size distribution of a large number of GO uniformly dispersed on the surfaces of $\mathrm{Al}$ flakes was measured and determined. An average in-plane size of $d$ $=0.5 \mu \mathrm{m}$ can be provided from the results in Fig. 2a. Fig. $2 \mathrm{~b}$ presents the irregular in-plane contour of single graphene with two basic parameters: the radius $R_{(\theta)}$ and angle $\theta$. In the structural model of graphene, the irregular inplane contour is modeled by varying radius $R_{(\theta)}$ and angle $\theta$ and the radius $R_{(\theta)}$ is determined by the average size $d$. Fig. $2 \mathrm{c}$ gives the 3D morphology construction of graphene by means of extruding the irregular in-plane contour of graphene with a distance $H$ along the $Z$-direction, in which the distance $H$ is equal to the thickness of graphene in this study. Fig. $2 \mathrm{~d}$ presents the meshed models of single graphene from different viewpoint in $X Y Z$ coordinate systems, where the in-plane contour and flat surface of graphene are presented. It is necessary for creating microscopic structural model of single graphene to further create the microscopic structural models of graphene/Al composites.

For generating 3D microscopic structural models of graphene/Al composites, we randomly distributed the graphene in $\mathrm{Al}$ matrix. Fig. 3a presents the basic flowchart about how to reproduce the actual composite structure of graphene/Al composites, where large amounts of graphene are dispersed with layered or dislayered structures. 


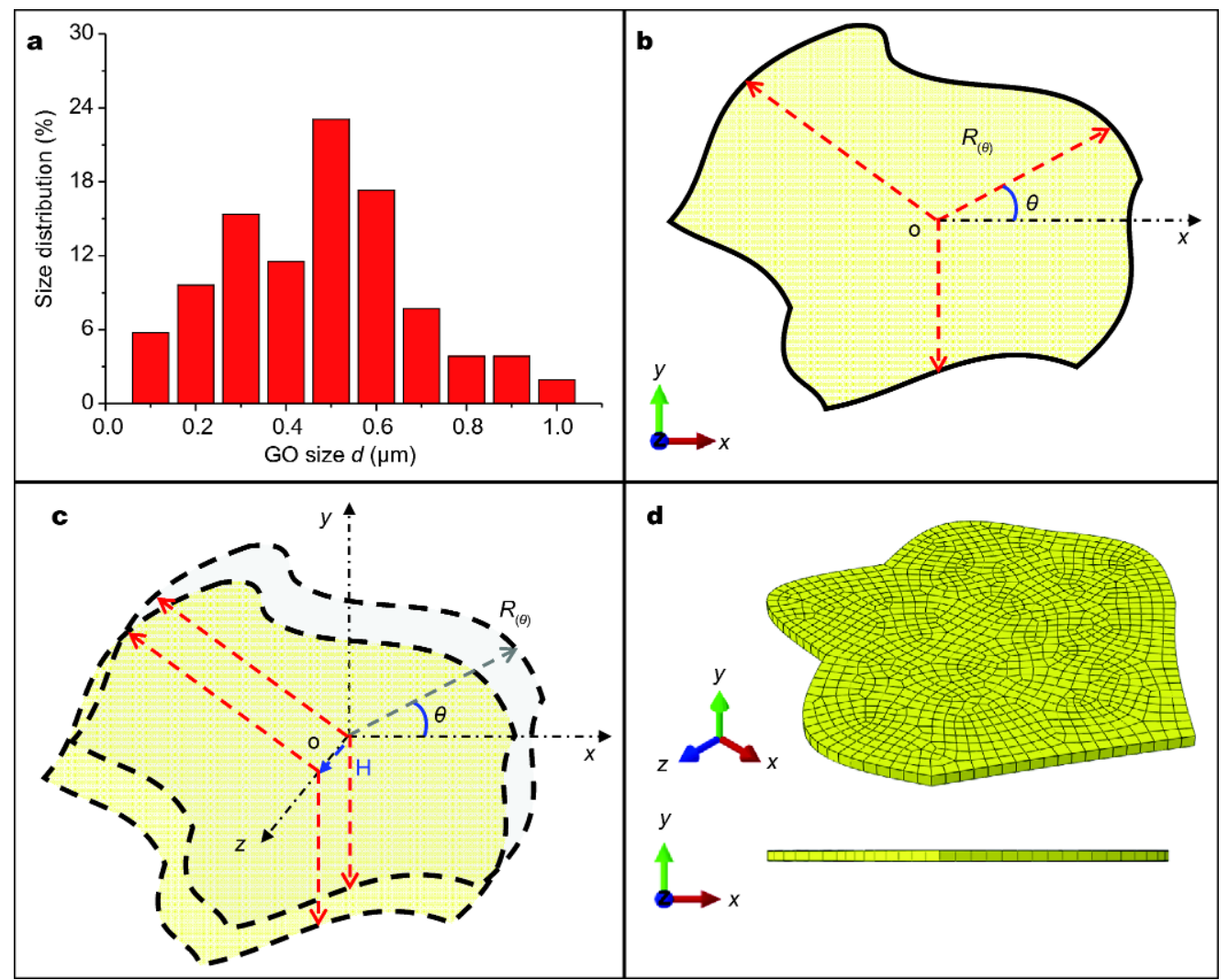

Figure 2 3D microscopic structural modeling of graphene reinforcement: (a) statistic size distribution of GO; (b) 2D in-plane contour of single graphene; (c) 3D structural modeling of single graphene and (d) 3D structural model of single graphene.

Fig. $3 \mathrm{~b}$ presents the microscopic structural model of single graphene, in which the average size $d$ and the thickness $H$ are based on the statistical results of size distribution of graphene. At the same time, the random factors such as the radius $f_{R}$, the angle $f_{\theta}$ and the thickness $f_{H}$ are used to reflect the structural variation of graphene. Fig. $3 \mathrm{c}$ presents the generating process by which multiple graphenes are randomly dispersed in the graphene/Al composites, where the graphenes are created one by one in order to avoid any overlay with the pre-generated graphenes. The increasing graphene content $V_{\mathrm{f}}$ is then compared with the desired graphene content $V_{\mathrm{fo}}$ in the actual graphene/Al composites. Once the desired graphene content $V_{\mathrm{fo}}$ has been obtained, the structural modeling process of the 3D microscopic structural models of the graphene/Al composites can be terminated. Therefore, the size, shape, orientation, location and content of graphene are reproduced in line with the actual graphene/Al composites. In order to form the 3D microscopic structural models of graphene/Al composites, a cubic representative structural model with a side length of $L$ can cut the dispersed graphene off. Fig. $3 \mathrm{~d}$ and e provide the $3 \mathrm{D}$ microscopic structural models (which own the side length of $L=5 h)$ of $1.5 \mathrm{vol} \%$ graphene/Al composite with layered structure (marked as 'LC') and dislayered structure (note as 'DC'), respectively. In fact, the independently developed Python-based structural modeling program is also able to establish the $3 \mathrm{D} \mathrm{mi-}$ croscopic structural models of graphene/Al composites with semi layered structure (labelled as 'SC'), in which both layered and dislayered graphene are dispersed in the composites). Due to the existence of random factors of single graphene, the size, shape, orientation, location and content of graphene in the microscopic structural models of graphene/Al composites are not simply repeated each time because of the difference of single graphene and the cutting location are not the same twice. The desired graphene content $V_{\mathrm{f}}$ can be precisely calculated by geometrical volume and controlled by the $3 \mathrm{D}$ structural modeling program. For the $\mathrm{Al}$ matrix and graphene/ $\mathrm{Al}$ composites, all the cubic structural models with the side length $L$ were applied on a uniaxial tensile displacement loading $U_{\mathrm{X}}=0.1 \mathrm{~L}$ along the $X$-axis direction and the general linear solid tetrahedron four-node elements 


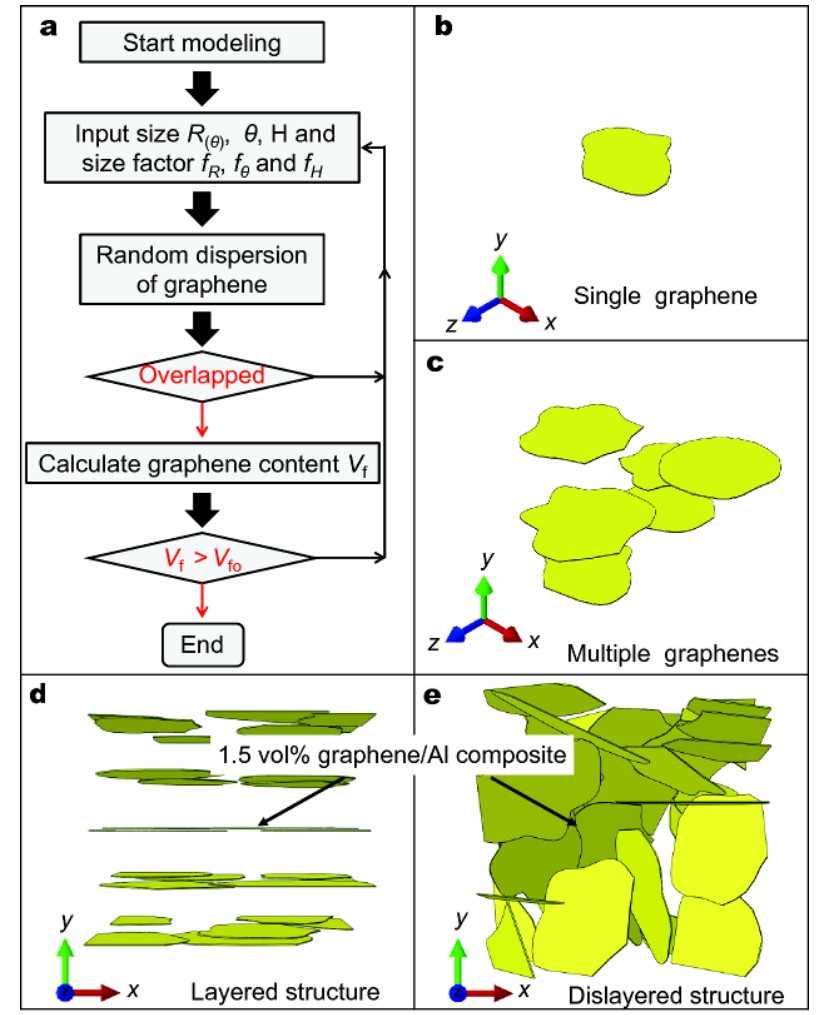

Figure 3 3D microscopic structural modeling of graphene/Al composites: (a) illustration of structural modeling; (b) structural model of single graphene; (c) structural model of multiple graphenes; structural models of 1.5 vol\% graphene/Al composites with (d) layered structure and (e) dislayered structure.

$\mathrm{C} 3 \mathrm{D} 4 \mathrm{H}$ and $\mathrm{C} 3 \mathrm{D} 4$ are used to mesh these structural models under free meshing technique.

\section{Mechanical properties of constituent materials in graphene/Al composites}

On basis of an analysis of literature data, the following mechanical properties of graphene and $\mathrm{Al}$ have been provided. For the graphene reinforcement and $\mathrm{Al}$ matrix, the bulk density $\rho$ are set to 2.25 and $2.7 \mathrm{~g} \mathrm{~cm}^{-3}$ [14], the elastic modulus $E$ are equal to $670 \mathrm{GPa}$ and $70 \mathrm{GPa}$ $[16,28]$, the Poisson's ratio $v$ are set to 0.186 and 0.33 [4], and the tensile strength $\sigma_{\text {UTS }}$ are equal to $63 \mathrm{GPa}$ and $200 \mathrm{MPa}$ [22], respectively, as shown in Fig. 4a. Fig. 4b provides the uniaxial tensile stress-strain relations of graphene reinforcement and pure $\mathrm{Al}$ matrix [22] in this analysis. Moreover, considering the existence of graphene in the graphene/ $\mathrm{Al}$ composites, the dislocations in the $\mathrm{Al}$ matrix can be hindered and piled up during the hotpressing and plastic deformation of graphene/Al composites. Therefore, due to the thermal mismatch between graphene and $\mathrm{Al}$, the stress increase of $\mathrm{Al}$ matrix can be written as [29]:

$$
\Delta \sigma=\alpha G b \rho^{1 / 2},
$$

where $\Delta \sigma$ is the stress increase of $\mathrm{Al}$ matrix, $\alpha=1.25$ is the geometric constant, $G=26.3 \mathrm{GPa}$ is the shear modulus, $b$ $=0.286 \mathrm{~nm}$ is the crystal constant of $\mathrm{Al}$ matrix, respectively. The dislocation density $\rho$ can be calculated by:

$$
\rho=\frac{B V_{\mathrm{f}} \varepsilon}{b\left(1-V_{\mathrm{f}}\right) t},
$$

where $B=8.0$ is for the plate reinforcement, $V_{\mathrm{f}}$ is the graphene content (vol\%) and $t=h$ is the smallest dimension and the thermal mismatch strain $\varepsilon$ can be defined as:

$$
\varepsilon=\Delta C T E \times \Delta T,
$$

where $\triangle C T E$ is the difference between the coefficients of thermal expansion of $\mathrm{Al}$ matrix $\left(23.6 \times 10^{-6} \mathrm{~K}^{-1}\right)$ and RGO $\left(-8.0 \times 10^{-6} \mathrm{~K}^{-1}\right)[30]$ and $\Delta T=500 \mathrm{~K}$ is the difference between the previous annealing and testing temperatures. From the equations above, the calculated dislocation density $\rho$ are around $0.167 \times 10^{14}$ and $0.336 \times$ $10^{14} \mathrm{~m}^{-2}$ for the 0.75 vol\% and 1.5 vol\% graphene/Al composites, respectively. Therefore, the stress increase of $\mathrm{Al}$ matrix in graphene/Al composites are 38.4 and $54.6 \mathrm{MPa}$ for the $0.75 \mathrm{vol} \%$ and $1.5 \mathrm{vol} \%$ graphene/Al composites (seen in Fig.4c), respectively. According to our previous work in Ref [31], we consider three basic graphene-Al interfacial behaviors: (i) adhesion interface; (ii) friction interface and (iii) cohesive interface in the simulations of mechanical deformation of graphene/Al composites. Graphene and $\mathrm{Al}$ matrix are always bonded for adhesion interface, and coefficient of friction 0.1 is presented for friction interface. Meanwhile, the cohesive zone model (CZM) can effectively express the interfacial damage behavior, in which the interfacial strength $\sigma_{0}$ and separation $\delta$ are usually specified, as shown in Fig. $4 \mathrm{~d}$. The interfacial damage behavior can be expressed and determined by the damage factor $D=\left(\left(\sigma_{\mathrm{n}} / \sigma_{0}\right)^{2}+\left(\sigma_{\mathrm{s}} / \sigma_{0}\right)^{2}\right)^{1 / 2}$. For the graphene-Al interface, the interfacial strength $\sigma_{0}$ is determined as $140 \mathrm{MPa}$, the fracture energy $\Gamma_{\mathrm{f}}$ is equal to $2 \mathrm{~J} \mathrm{~m}^{-2}$, respectively $[32,33]$. In order to simulate the fracture mechanical behavior of $\mathrm{Al}$ matrix and graphene/ $\mathrm{Al}$ composites, the ductile damage criterion (in which the fracture strain $\varepsilon_{\mathrm{f}}$ and displacement at fracture $u_{\mathrm{f}}$ are usually applied) is applied for both graphene reinforcement and $\mathrm{Al}$ matrix, respectively. In this analysis, the fracture strain $\varepsilon_{\mathrm{f}}$ is set to 0.09 and 0.06 separately, and displacement at failure $u_{\mathrm{f}}$ is determined as $1 \mathrm{~nm}$ and $1 \mathrm{~nm}$, respectively. The numerical simulations have been performed using the commercial finite element code Abaqus. 


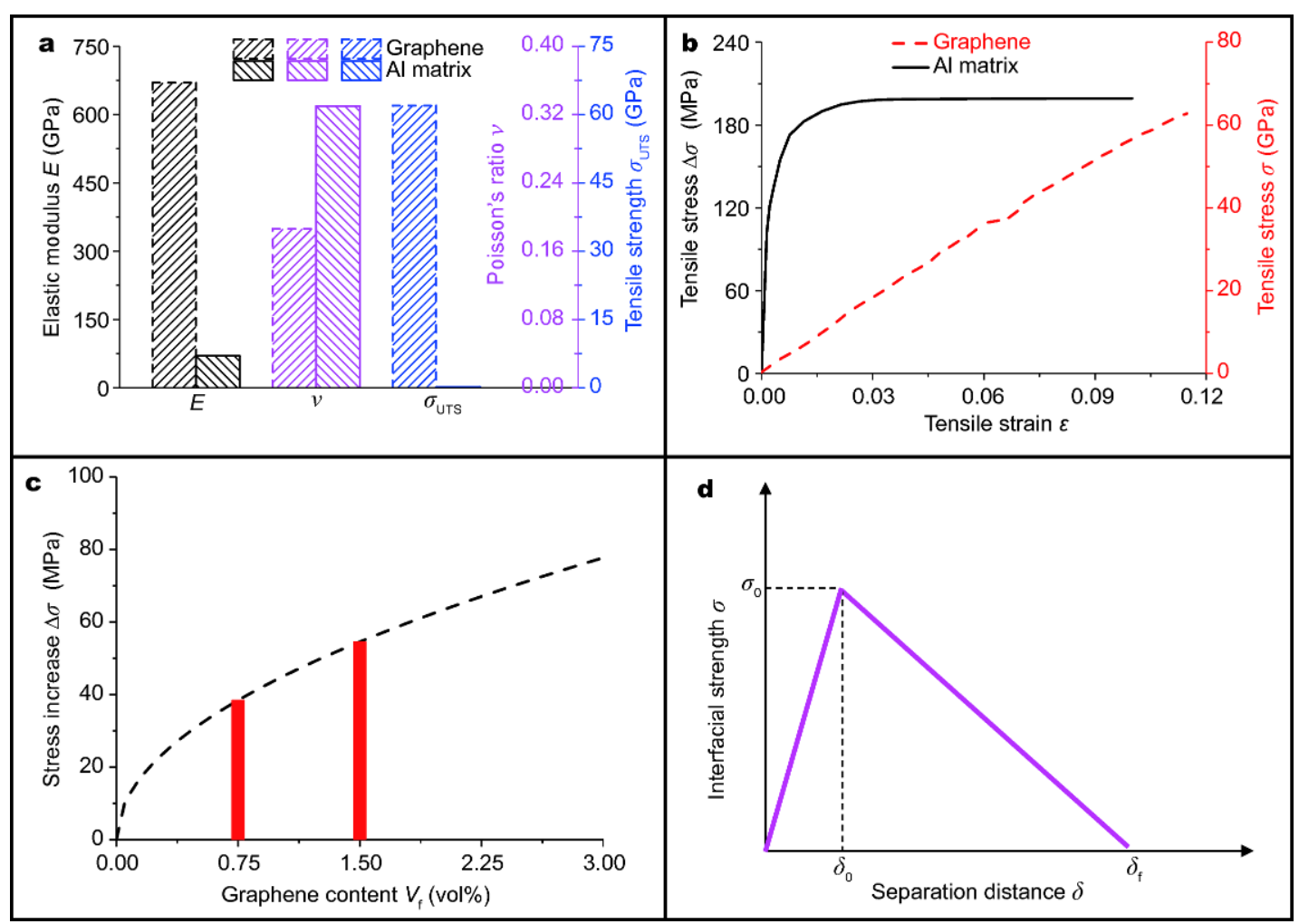

Figure 4 Mechanical properties and interfacial behavior in graphene/Al composites: (a) elastoplastic mechanical properties; (b) tensile stress-strain relations; (c) strengthening stress increase in $\mathrm{Al}$ matrix and (d) damaged cohesive interfacial model of graphene- $\mathrm{Al}$ interfaces.

\section{RESULTS AND DISCUSSION}

Effects of mesh size, failure behavior, model validity and model size on tensile properties of $\mathrm{Al}$ matrix and graphene/Al composites

From the obtained simulating results of $\mathrm{Al}$ matrix and graphene/Al composites, the tensile stress-strain relations can be determined. For each 3D microscopic structural model of $\mathrm{Al}$ matrix or graphene/Al composites with a certain graphene content, the tensile stress and strain can be calculated by the reaction force $R F_{\mathrm{X}}$ and the displacement $U_{\mathrm{X}}$ divided by the initial contact area $L \times L$ and initial length $L$. Due to the definition above, the numerical tensile stress-strain relations of $\mathrm{Al}$ matrix and graphene/Al composites can be derived. Fig. 5a shows a good accordance between the experimental and numerical tensile stress-strain relations of $\mathrm{Al}$ matrix with different mesh size. In this study, the side length of cubic structural model of $\mathrm{Al}$ matrix is selected as $L=3 h$ (that is equal to $645 \mathrm{~nm}$ ). The mesh sizes are separately considered as 50, $40,30,20$ and $10 \mathrm{~nm}$, while the number of meshed elements are 13,502, 23,088, 53,196, 137,595 and 698,488, respectively. Considering the balance of computing ac- curacy and computing cost, the mesh size of $20 \mathrm{~nm}(\sim h /$ $10)$ is selected and preferred in all the other simulations for both $\mathrm{Al}$ matrix and graphene/Al composites. Fig. 5b presents a good agreement between the experimental and numerical tensile stress-strain relations of $\mathrm{Al}$ matrix without and with damage materials behavior. Through the adjustment of the ductile damage parameters (e.g. the fracture strain $\varepsilon_{\mathrm{f}}$ and displacement at fracture $u_{\mathrm{f}}$ ) and the comparison between experimental and numerical tensile stress-strain relations of $\mathrm{Al}$ matrix, the suitable values of $\varepsilon_{\mathrm{f}}$ $=0.09$ and $u_{\mathrm{f}}=1 \mathrm{~nm}$ are selected and preferred to simulate the failure behavior of $\mathrm{Al}$ matrix and graphene/Al composites.

Fig. $5 c$ presents six different numerical tensile stressstrain relations of 0.75 vol\% graphene/Al composites with layered structures and cohesive interfaces. It should be noted that just the shapes and locations of graphene are differing from each other in the structural models of graphene/Al composites during each simulation, that is to say, they are not other simply repetitive structural models of the same one, but six simulations of different structural models of graphene/Al composites with the same graphene content $(0.75 \mathrm{vol} \%)$. Although the microscopic 


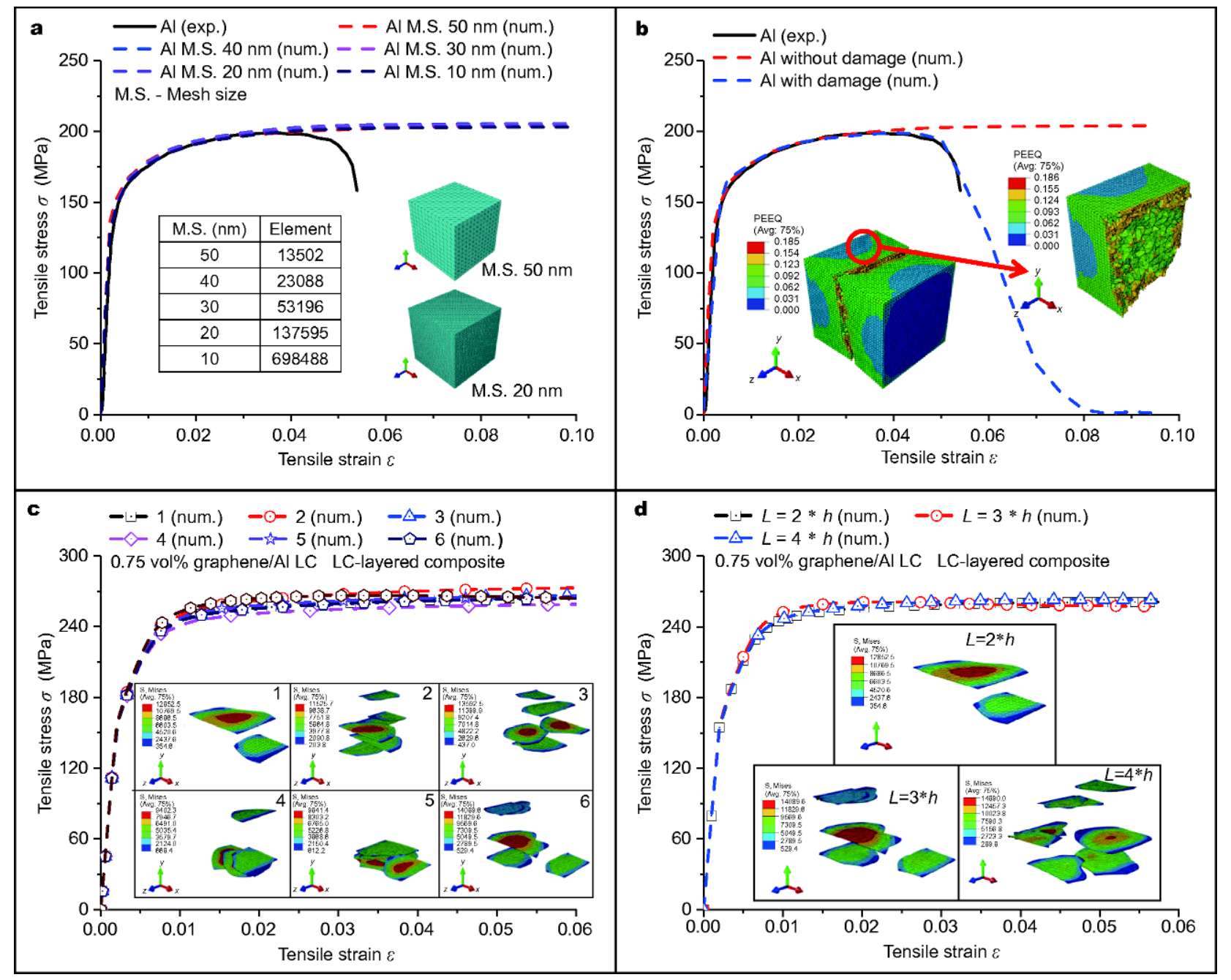

Figure 5 Tensile mechanical properties and equivalent strain or stress distributions in Al matrix with: (a) different mesh size and (b) failure materials behavior; and in 0.75 vol\% graphene/Al composites with: (c) model repeatability and (d) different model size.

structural models of $0.75 \mathrm{vol} \%$ graphene/Al composites are different, all the numerical tensile stress-strain relations obtained just generate a small diversity at the whole tensile strain, in which the tensile strength $\sigma_{\mathrm{UTS}}$ is 267.1 $\pm 7.0 \mathrm{MPa}$ at the tensile strain $\varepsilon=0.06$. Meanwhile, the largest equivalent stress within the graphene reinforcement is no more than $15.0 \mathrm{GPa}$, which indicates that the graphene may not have completely exhibited the strengthening effect on the tensile mechanical properties of graphene/Al composites. Fig. 5d also compares the numerical tensile stress-strain relations of $0.75 \mathrm{vol} \%$ graphene/Al composites with layered structures and cohesive interfaces, in which the different side length of cubic structural model is selected as $L=2 h, 3 h$ and $4 h$. For the three different model sizes, the numerical tensile stress-strain relations of $0.75 \mathrm{vol} \%$ graphene/Al compo- sites generally agree with each other. These results means that the preferred model size of $L=3 h$ can be selected and applied for the following simulations of graphene/Al composites on balance of the computing accuracy and computing cost.

\section{Effects of interfacial behavior and composite configuration on tensile properties of graphene/Al composites}

Fig. $6 \mathrm{a}, \mathrm{b}$ present experimental and numerical tensile stress-strain relations of 0.75 vol\% and 1.5 vol\% graphene/Al composites with three different interfacial behaviors: adhesion, cohesive and friction interfaces, respectively. From the numerical results, the tensile stress-strain relation of the graphene/Al composite with adhesion interface presents the largest stresses within the entire tensile strain region, while the tensile stress-strain 


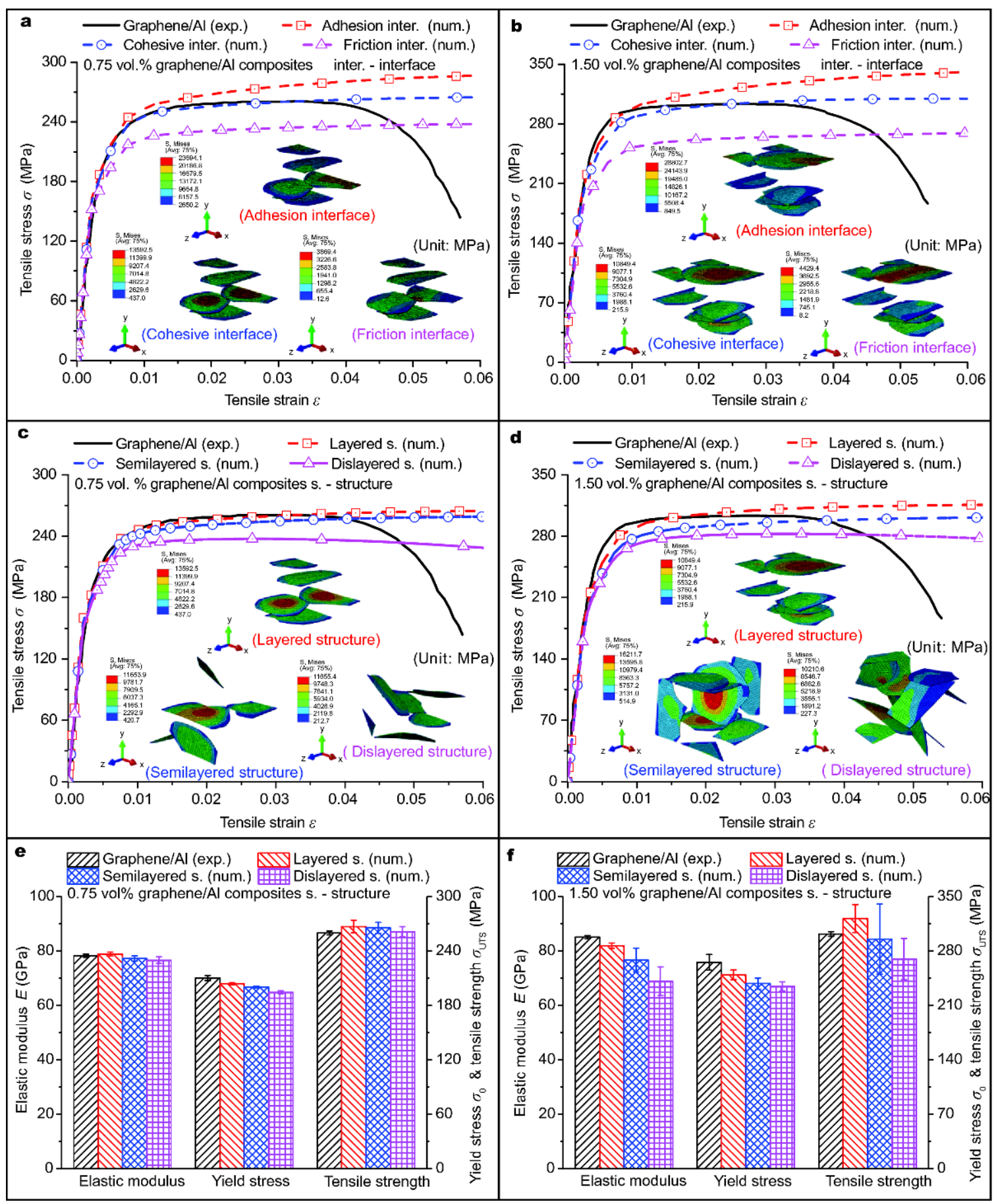

Figure 6 Tensile mechanical properties and equivalent stress distributions of graphene/Al composites with differing interfacial behaviors for: (a) 0.75 vol\% and (b) $1.50 \mathrm{vol} \%$ graphene/Al composites; with varying composite configurations for: (c), (e) $0.75 \mathrm{vol} \%$ and (d), (f) $1.50 \mathrm{vol} \%$ graphene/Al composites, respectively.

relation of the graphene/Al composite with friction interface produces the lowest stresses over the whole tensile strain region. Meanwhile, the tensile stress-strain relation of the graphene/Al composite with cohesive interface provides the closest result to the experimental ones of both $0.75 \mathrm{vol} \%$ and $1.5 \mathrm{vol} \%$ graphene/Al composites. However, it should be noted that the tensile stresses of both $0.75 \mathrm{vol} \%$ and $1.5 \mathrm{vol} \%$ graphene/Al composites 
with adhesion, cohesive and friction interfaces generate a large difference to each other, in which the tensile stresses (at the tensile strain $\varepsilon=0.06$ ) change from 287.2 to $238.1 \mathrm{MPa}$, from 341.6 to $269.5 \mathrm{MPa}$ and the maximum equivalent stresses in graphene vary from 23.7 to $3.8 \mathrm{GPa}$, from 28.8 to $4.4 \mathrm{GPa}$ for the $0.75 \mathrm{vol} \%$ and $1.5 \mathrm{vol} \%$ graphene/Al composites, respectively. The analysis above indicates that the graphene content (vol\%) and the graphene-Al interfacial behavior play the significant roles in enhancing the mechanical properties of graphene/Al composites.

Fig. $6 \mathrm{c}$ and $\mathrm{d}$ provide experimental and numerical tensile stress-strain relations of $0.75 \mathrm{vol} \%$ and $1.5 \mathrm{vol} \%$ graphene/Al composites with three different composite configurations: layered, semilayered and dislayered structures, respectively. It should be pointed out that just the $X$-axis loading direction is conducted in all the simulations, in which the loading direction is parallel to the in-plane layer for the graphene/Al composite with layered structure. The tensile stress-strain relation of the graphene/Al composite with layered structure presents the largest stresses over the entire tensile strain region, while the tensile stress-strain relation of the graphene/Al composite with dislayered structure yields the lowest stresses over the whole tensile strain region. Meanwhile, the tensile stress-strain relation of the graphene/Al composite with layered structure presents the closest result to the experimental ones of the actual $0.75 \mathrm{vol} \%$ and $1.5 \mathrm{vol} \%$ graphene/Al composites with layered structure. Meanwhile, it is worth noting that the tensile stresses of both 0.75 vol\% and 1.5 vol\% graphene/Al composites with the layered, semilayered and dislayered structure produce a large variation to each other, in which the tensile stresses (at the tensile strain $\varepsilon=0.06$ ) range from 265.1 to $228.1 \mathrm{MPa}$, from 315.8 to $277.1 \mathrm{MPa}$ and the maximum equivalent stresses in graphene differ from 13.6 to $11.7 \mathrm{GPa}$, from 10.8 to $10.2 \mathrm{GPa}$. These simulating results mean that the graphene content (vol\%) again and composite configuration play the important roles in enhancing the mechanical properties of graphene/ $\mathrm{Al} \mathrm{com-}$ posites.

Fig. 6e and $\mathrm{f}$ provide experimental and numerical tensile mechanical properties (which contain the elastic modulus, yield stress and tensile strength, respectively) of $0.75 \mathrm{vol} \%$ and $1.5 \mathrm{vol} \%$ graphene/Al composites with three different composite configurations: layered, semilayered and dislayered structures, respectively. For numerical tensile deformations of graphene/Al composites with each graphene content and each composite configuration, 10 different structural modeling and properties calculations are performed and repetitive in order to provide the statistic tensile mechanical properties of graphene/Al composites. Meanwhile, for the tensile deformations of $0.75 \mathrm{vol} \%$ and $1.5 \mathrm{vol} \%$ graphene/Al composites with layered structures, three different tensile tests are carried out for each case. The results show that the experimental and simulated tensile mechanical properties of $0.75 \mathrm{vol} \%$ graphene/Al composites with different composite configurations produce a little difference between each other. Meanwhile, all the tensile mechanical properties of $0.75 \mathrm{vol} \%$ graphene/Al composites generate a slight decreasing tendency with the composite configuration ranging from the layered, to the semilayered, to the dislayered structures, respectively. For $1.5 \mathrm{vol} \%$ graphene/Al composites, the experimental elastic modulus and yield stress are relatively larger than those of numerical results, while the experimental tensile strength is equivalent to those of numerical simulations. At the same time, for each numerical tensile mechanical property, a relatively larger declining tendency exists with the composite configuration changing from the layered, to the semilayered, and to the dislayered structures, respectively. This analysis indicates that the composite configuration, e.g. the distributed means of graphene in $\mathrm{Al}$ matrix, plays a key role in the tensile mechanical properties of graphene/ $\mathrm{Al}$ composites due to the diversity of composite structure and materials property.

\section{Effect of material failure behavior on tensile deformations of graphene/Al composites}

Fig. $7 \mathrm{a}, \mathrm{b}$ provide experimental and numerical tensile stress-strain relations of $0.75 \mathrm{vol} \%$ and $1.5 \mathrm{vol} \%$ graphene/ $\mathrm{Al}$ composites with layered structures, in which the basic damage behaviors of graphene, $\mathrm{Al}$ matrix and graphene-Al interface are applied in the simulations. For the 0.75 vol\% and 1.5 vol\% graphene/Al composites, we can see that the numerical tensile stress-strain relations are generally close to those of experimental results, even for the elastic and plastic stage. As for the damage stage, a little large difference exists between the numerical and experimental stress-strain relations. Moreover, the equivalent strain distributions of $0.75 \mathrm{vol} \%$ and $1.5 \mathrm{vol} \%$ graphene/ $\mathrm{Al}$ composites corresponding to simulated models are presented as well, in which the cracks are exhibited within the failure behavior of graphene/Al composites. Compared to the experimental tensile fracture section of $0.75 \mathrm{vol} \%$ graphene/Al composite, Fig. $7 \mathrm{c}$ shows the virtual 3D deformed structural model (that owns the model size of $3 h \times 3 h \times 3 h)$ after the fracture failure, and the related numerical tensile stress-strain 


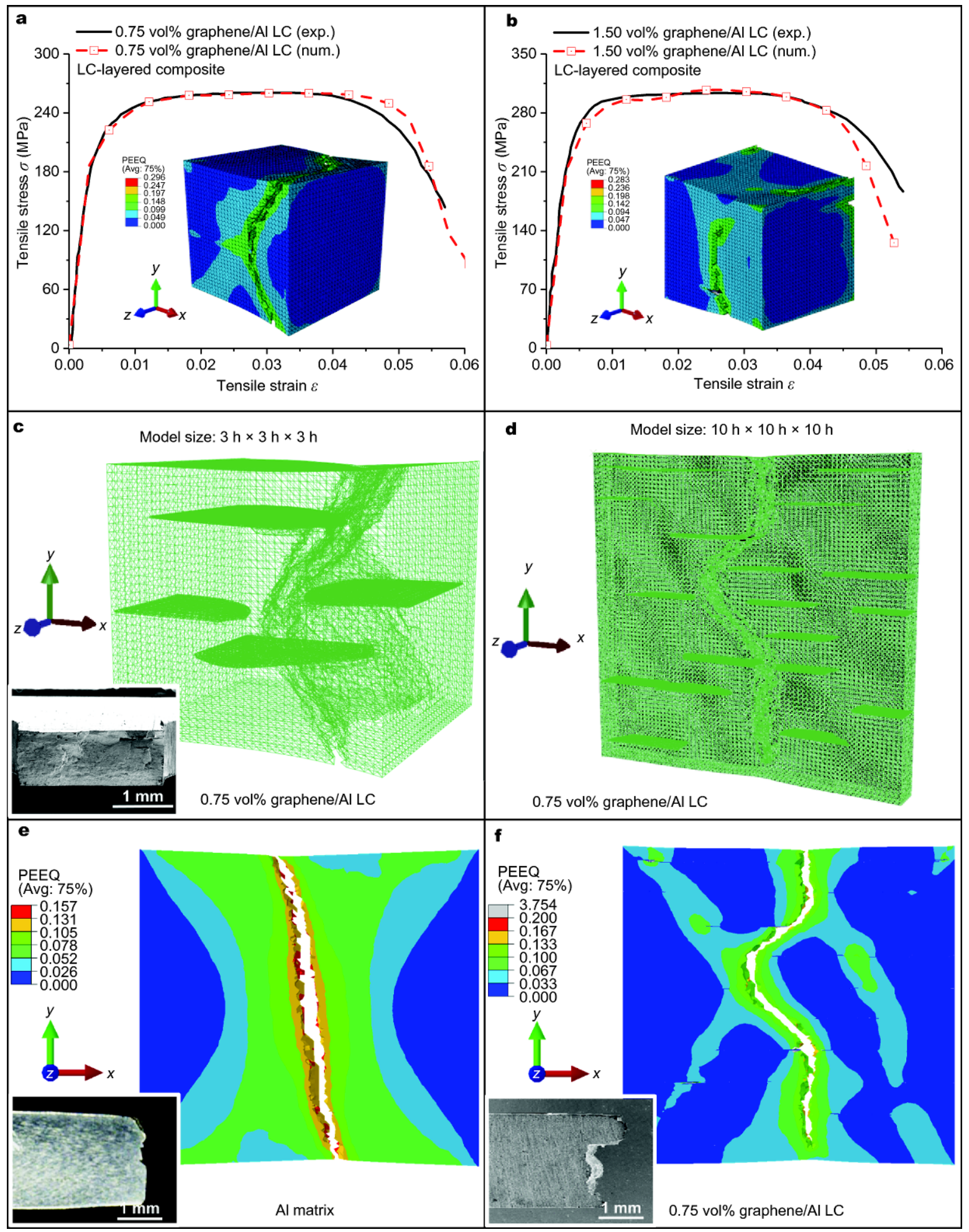

Figure 7 Tensile mechanical properties and equivalent strain distributions of graphene/Al composites with failure behaviors for: (a) 0.75 vol\% and (b) $1.50 \mathrm{vol} \%$ graphene/Al composites; Virtual tensile crack propagations in $0.75 \mathrm{vol} \%$ graphene/Al composites with the structural model size of (c) $3 h \times 3 h \times 3 h$ and (d) $10 h \times 10 h \times h$; Tensile crack growth paths at the failure of (e) Al matrix and (f) 0.75 vol\% graphene/Al composites, respectively. 
relation is provided in Fig. 7a. What we can see is that the crack location mainly situates within the $\mathrm{Al}$ matrix, and the cracks usually initiate within the graphene-Al interfacial area. Moreover, the crack propagation path is zigzag but not straight within the tensile fracture samples. At the same time, we have not found the fracture failure in the graphene, which may be attributed to the relatively weak interfacial strength $(\sim 140 \mathrm{MPa})$ to fracture strength of graphene $(\sim 63 \mathrm{GPa})$.

In order to further indicate the fracture propagation in graphene/Al composites, the virtual 3D deformed structural model (that owns the model size of $10 h \times 10 h \times h$ ) of $0.75 \mathrm{vol} \%$ graphene/Al composite after the fracture failure is also presented in Fig. $7 \mathrm{~d}$. Once again, the crack mainly locates within the $\mathrm{Al}$ matrix, and the cracks usually produce within the graphene-Al interfacial areas. The crack propagation has been buckled as a result of the existing of graphene reinforcement. Fig. 7e, $\mathrm{f}$ provide the equivalent strain distributions in $\mathrm{Al}$ matrix and $0.75 \mathrm{vol} \%$ graphene/Al composite, in which the crack propagation paths both in experimental samples and simulated models are relatively straight in pure $\mathrm{Al}$ matrix and flexuous in graphene/Al composite due to the existence of the graphene reinforcement. Meanwhile, the fracture locations mostly situate at the $\mathrm{Al}$ matrix near the graphene- $\mathrm{Al}$ interfaces and the intensive dispersion of graphene, while all the graphene reinforcements hardly fracture during the tensile deformation of $0.75 \mathrm{vol} \%$ graphene/Al composite. Otherwise, the tensile failure behavior of graphene/Al composites is successfully simulated, which reveals the roles of graphene, $\mathrm{Al}$ matrix and graphene- $\mathrm{Al}$ interface in the failure behavior of graphene/Al composites.

\section{CONCLUSIONS}

In this analysis, the 3D microstructural modeling, experimental investigation and numerical simulation of uniaxial tensile mechanical deformation of graphene/Al composites were performed. In the numerical work, the strengthening property, interfacial behavior, composite configuration and fracture behavior were incorporated. Several conclusions can be drawn as following:

(i) Based on the microscopic structural characteristics of the constituent materials in graphene/ $\mathrm{Al}$ composites, the distributions of graphene in-plane size and $\mathrm{Al}$ layer thickness are statistically summarized. The average sizes and random factors are applied to construct 3D microscopic structural model of single graphene, in which inplane contour, thickness and orientation of graphene can be varied. Furthermore, an independently developed Py- thon-based structural modeling program can effectively establish the 3D microscopic structural models of graphene/Al composites, in which the size, shape, orientation, location and content of graphene can be reproduced to reflect the actual composite structures of graphene/Al composite products.

(ii) Experimental uniaxial tensile tests of graphene/Al composites with differing graphene content were performed. Combing the strengthened matrix properties and damaged graphene-Al interfacial behavior, a series of numerical tensile deformations of graphene/Al composites with different graphene content, interfacial behavior and composite configuration are carried out on the created structural models. On basis of the numerical results, it indicates that the graphene content, the graphene- $\mathrm{Al}$ interfacial behavior and composite configuration generally play the significant roles in enhancing the mechanical properties of graphene/ $\mathrm{Al}$ composites.

(iii) By introducing the failure behavior of constituent materials in graphene/Al composites, the fracture behavior of graphene/Al composites can be simulated as well. The materials failure or generated cracks mostly situates within the $\mathrm{Al}$ matrix that are close to the graphene/Al composites, while the crack path is relatively flexuous due to the existence of graphene distributed in Al matrix. Generally speaking, the conducted numerical investigating method in this work can be applied to effectively establish the relationship between the composite structure and mechanical property within the graphene-based composites.

Received 27 July 2017; accepted 15 October 2017; published online 28 November 2017

1 Geim AK, Novoselov KS. The rise of graphene. Nat Mater, 2007, 6: 183-191

2 Novoselov KS, Fal'ko VI, Colombo L, et al. A roadmap for graphene. Nature, 2012, 490: 192-200

3 Daniels C, Horning A, Phillips A, et al. Elastic, plastic, and fracture mechanisms in graphene materials. J Phys-Condens Matter, 2015, 27: 373002

4 Liu F, Ming P, Li J. Ab initio calculation of ideal strength and phonon instability of graphene under tension. Phys Rev B, 2007, 76: 064120

5 Lee C, Wei X, Kysar JW, et al. Measurement of the elastic properties and intrinsic strength of monolayer graphene. Science, 2008, 321: $385-388$

6 Balandin AA. Thermal properties of graphene and nanostructured carbon materials. Nat Mater, 2011, 10: 569-581

7 Morozov SV, Novoselov KS, Katsnelson MI, et al. Giant intrinsic carrier mobilities in graphene and its bilayer. Phys Rev Lett, 2008, 100: 016602

8 Porwal H, Grasso S, Reece MJ. Review of graphene-ceramic matrix composites. Adv Appl Ceramics, 2013, 112: 443-454 
9 Das TK, Prusty S. Graphene-based polymer composites and their applications. Polymer-Plastics Tech Eng, 2013, 52: 319-331

10 Tjong SC. Recent progress in the development and properties of novel metal matrix nanocomposites reinforced with carbon nanotubes and graphene nanosheets. Mater Sci Eng-R-Rep, 2013, 74: 281-350

11 Rafiee MA, Rafiee J, Wang Z, et al. Enhanced mechanical properties of nanocomposites at low graphene content. ACS Nano, 2009, 3: 3884-3890

12 Wan C, Chen B. Reinforcement and interphase of polymer/graphene oxide nanocomposites. J Mater Chem, 2012, 22: 3637-3646

13 Chen LY, Konishi H, Fehrenbacher A, et al. Novel nanoprocessing route for bulk graphene nanoplatelets reinforced metal matrix nanocomposites. Scripta Mater, 2012, 67: 29-32

14 Lin D, Richard Liu C, Cheng GJ. Single-layer graphene oxide reinforced metal matrix composites by laser sintering: Microstructure and mechanical property enhancement. Acta Mater, 2014, 80: 183-193

15 Chu K, Jia C. Enhanced strength in bulk graphene-copper composites. Phys Status Solidi A, 2014, 211: 184-190

16 Liu L, Zhang J, Zhao J, et al. Mechanical properties of graphene oxides. Nanoscale, 2012, 4: 5910-5916

17 Dikin DA, Stankovich S, Zimney EJ, et al. Preparation and characterization of graphene oxide paper. Nature, 2007, 448: 457-460

18 Park S, Ruoff RS. Chemical methods for the production of graphenes. Nat Nanotech, 2009, 4: 217-224

19 Avouris P, Dimitrakopoulos C. Graphene: synthesis and applications. Mater Today, 2012, 15: 86-97

20 Montazeri A, Rafii-Tabar H. Multiscale modeling of graphene- and nanotube-based reinforced polymer nanocomposites. Phys Lett A, 2011, 375: 4034-4040

21 Suk JW, Piner RD, An J, et al. Mechanical properties of monolayer graphene oxide. ACS Nano, 2010, 4: 6557-6564

22 Paci JT, Belytschko T, Schatz GC. Computational studies of the structure, behavior upon heating, and mechanical properties of graphite oxide. J Phys Chem C, 2007, 111: 18099-18111

23 Parashar A, Mertiny P. Multiscale model to investigate the effect of graphene on the fracture characteristics of graphene/polymer nanocomposites. Nanoscale Res Lett, 2012, 7: 595

24 Dai G, Mishnaevsky Jr. L. Graphene reinforced nanocomposites: 3D simulation of damage and fracture. Comp Mater Sci, 2014, 95: 684-692
25 Azoti WL, Elmarakbi A. Multiscale modelling of graphene platelets-based nanocomposite materials. Composite Struct, 2017, 168: 313-321

26 Jiang L, Li Z, Fan G, et al. The use of flake powder metallurgy to produce carbon nanotube (CNT)/aluminum composites with a homogenous CNT distribution. Carbon, 2012, 50: 1993-1998

27 Li Z, Fan G, Tan Z, et al. Uniform dispersion of graphene oxide in aluminum powder by direct electrostatic adsorption for fabrication of graphene/aluminum composites. Nanotechnology, 2014, 25: 325601

28 Young RJ, Kinloch IA, Gong L, et al. The mechanics of graphene nanocomposites: a review. Composites Sci Tech, 2012, 72: 1459_ 1476

29 Arsenault RJ, Shi N. Dislocation generation due to differences between the coefficients of thermal expansion. Mater Sci Eng, 1986, 81: 175-187

30 Yoon D, Son YW, Cheong H. Negative thermal expansion coefficient of graphene measured by Raman spectroscopy. Nano Lett, 2011, 11: 3227-3231

31 Su Y, Li Z, Jiang L, et al. Computational structural modeling and mechanical behavior of carbon nanotube reinforced aluminum matrix composites. Mater Sci Eng-A, 2014, 614: 273-283

32 Liu Z, Zhang SM, Yang JR, et al. Interlayer shear strength of single crystalline graphite. Acta Mech Sin, 2012, 28: 978-982

33 Feng S, Guo Q, Li Z, et al. Strengthening and toughening mechanisms in graphene-Al nanolaminated composite micro-pillars. Acta Mater, 2017, 125: 98-108

Acknowledgements The authors acknowledge the financial supports by the National Natural Science Foundation (51501111, 51131004), the Ministry of Science and Technology of China (2016YFE0130200), Science \& Technology Committee of Shanghai (14DZ2261200, 1452 0710100 and 14JC14033 00) and 111 Project (B16032).

Author contributions Zhang D and Su Y designed and directed the overall study. Sample preparation, tensile tests and SEM, TEM observation were carried out by Li Z, Yu Y and Zhao L, respectively. Su Y wrote the manuscript and discussed the results and analyzed the data with Li ZQ, Guo Q and Xiong D.

Conflict of interest The authors declare that they have no conflict of interest. 

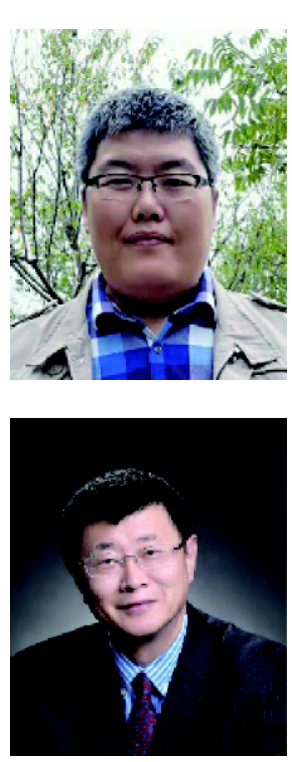

Yishi Su received his PhD from University of Technology of Troyes, France in 2012, and joined Professor Di Zhang's group as a post-doctor and assistant professor at Shanghai Jiao Tong University since 2012, 2014. His research interests focus on biomimetic metal matrix composites and materials genome computation.
Di Zhang received his $\mathrm{PhD}$ from Osaka University, Japan. He is now a Chair Professor of Materials Science and Engineering at Shanghai Jiao Tong University in China (since 1994), the director of State Key Lab of Metal Matrix Composites and the Institute of Composite Materials at SJTU (since 2003), and the Professor of Chang Jiang Scholars Program (since 2001). Prof. Zhang has published more than 200 peer reviewed academic articles, 1 English academic book on morphology-genetic materials, and attended international conferences as invited speakers for 47 times. His research interests include the process of advanced metal matrix composites and the basic and applied research on biomimetic morphology-genetic materials.

\section{石墨烯增强金属基复合材料的结构建模与拉伸模拟}

苏益士, 李赞, 俞洋, 赵蕾, 李志强, 郭强, 熊定邦, 张荻 ${ }^{*}$

摘要 石墨烯具有优异的机械性能, 已成为众多复合材料中的理想增强体材料. 本研究采用片状粉末冶金方法制备了具有仿生叠层结构 的石墨烯/铝基复合材料, 同时对纯铝基体与石墨烯/铝基复合材料进行了拉伸试验. 通过基于Python语言自主研发的复合材料结构建模 程序, 可以有效建立石墨烯/铝基复合材料的三维复合结构模型, 并实现石墨烯尺寸、形貌、取向、位置与含量等可控重构分布. 通过引 入组分材料力学性能、损伤行为、界面行为及边界条件等实现了石墨烯/铝基复合材料的拉伸行为模拟, 并揭示了石墨烯形貌、石墨烯/ 铝界面、复合构型与失效行为等复合因素的影响规律, 对理解石墨烯/铝基复合材料的变形机理提供了有力依据. 